\title{
Tuberculous Myopericarditis: an Incidental Finding on Autopsy
}

\author{
Veena Gupta1, Nidhi Kaushik ${ }^{2}$, Sucheta ${ }^{3 *}$, Rajnish Kalra ${ }^{1}$, Raman Kapil ${ }^{2}$ and Rajeev Sen ${ }^{4}$ \\ ${ }^{1}$ Professor, Department of Pathology, Pt. B. D. Sharma PGIMS, Rohtak, Haryana, India \\ ${ }^{2} J u n i o r$ Resident, Department of Pathology, Pt. B. D. Sharma PGIMS, Rohtak, Haryana, India \\ ${ }^{3}$ Senior Resident, Department of Pathology, Pt. B. D. Sharma PGIMS, Rohtak, Haryana, India \\ ${ }^{4}$ Senior Professor and Head of Department, Department of Pathology, Pt. B. D. Sharma PGIMS, Rohtak, Haryana, India \\ *Corresponding Author: Sucheta Department of Pathology, Pt. B. D. Sharma PGIMS Rohtak, Haryana, India
}

Received: July 01, 2019; Published: July 18, 2019

DOI: 10.31080/ASMS.2019.03.0361

\begin{abstract}
Tuberculosis is an important infectious condition in many developing countries especially India. But sometimes its detection can be late when it affects organs other than those that are commonly involved. Here we report case of a deceased person who had a history of fever and cough for which the patient did not consult a doctor and was taking home remedies and self medication only. Later he was diagnosed with tuberculous myopericarditis on autopsy. This case depicts an excellent example of how a very common pathological condition like tuberculosis can present in uncommon ways that can lead to delay in diagnosis.
\end{abstract}

Keywords: Autopsy; myopericarditis; tuberculosis

\section{Introduction}

Tuberculosis is an important health problem especially in countries like India. It spares few organs in body including thyroid, heart, pancreas and skeletal muscle [1]. Cardiac involvement is usually the result of dissemination from lesions elsewhere in the body. The pericardium is most commonly involved in cardiac tuberculosis [2]. The earliest case of myocardial tuberculosis was reported in 1664 by Maurocordat, a Turkish physician [3]. However, the myocardium was not recognized as a specific site of Mycobacterium tuberculosis infection till late centuries.

Tuberculous involvement of myocardium is rare, and the prevalence of the reported isolated myocardial tuberculosis is $0.14 \%, 0$. $2 \%$ and $2 \%$ in various series [4] and it generally occurs in conjunction with pericardial involvement.

The myocardium may be affected by direct spread from mediastinal lymph nodes or tuberculous pericarditis, as part of miliary tuberculosis, or as a result of retrograde lymphatic and hematogenous spread [5,6].Antemortem diagnosis of tubercular myocarditis is rare due to lack of awareness and knowledge of disease in developing countries like India.

\section{CASE REPORT}

We present a case report of tuberculous myopericarditis, from a tertiary care center of north india, diagnosed on post mortem histopathological examination of heart. The deceased was 51years old male who died due to heart attack according to relatives. The relatives of the patient also gave history of fever and cough for which the patient did not consult a doctor and was taking home remedies and self medication only. The deceased did not have any other significant history except that he was a chronic alcoholic.

Autopsy was performed on the same day of patient's death. The deceased was thinly built and poorly nourished. Various organs including heart, liver and lung were sent for histopathological examination. The heart weighted $650 \mathrm{gm}$ and measured $10 \times 10 \times 6 \mathrm{~cm}$. External surface of heart showed multiple grey white nodules. (Figure 1) On cutting open, right ventricular wall and left ventricular wall measured $0.4 \mathrm{~cm}$ and $1.4 \mathrm{~cm}$ in thickness and showed few grey white areas. Multiple grey white areas were also seen in lung and liver. On microscopic examination, sections from heart revealed thickening of pericardium and myocardium alongwith presence of caseous necrosis, epithelioid cell granulomas and mononuclear cell infiltrate (Figure 3). Similar changes were seen on histopathology of lung and liver also (Figure 2). Ziehl-Neelsen staining for acid fast bacilli using $20 \% \mathrm{H}_{2} \mathrm{SO}_{4}$ was also positive in sections from heart, lung and liver (Figure 4,5). PAS stain was also performed that came out to be negative. Antemortem diagnosis of tuberculosis was not made in this case. Other organs involvement revealing necrotizing epithelioid cell granulomas indicate that pericardium and myocardium could be involved as a result of dissemination following a primary tuberculosis most probably of lung. 


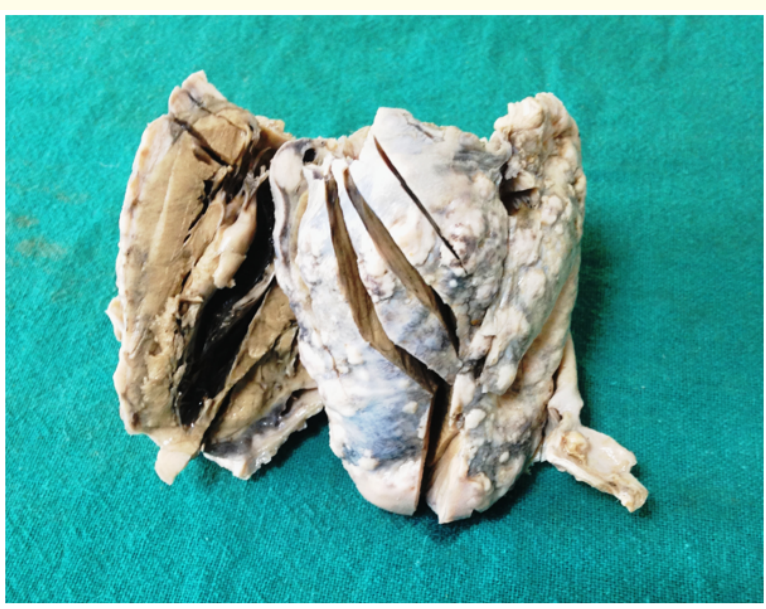

Figure 1: External surface of heart showed multiple grey white nodules.

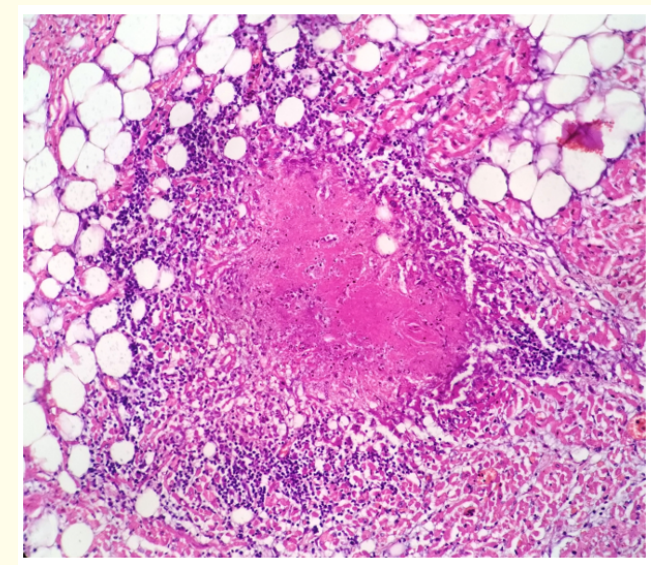

Figure 2: Microsections from heart revealing caseous necrosis, epithelioid cell granulomas and mononuclear cell infiltrate. (HE;200x)

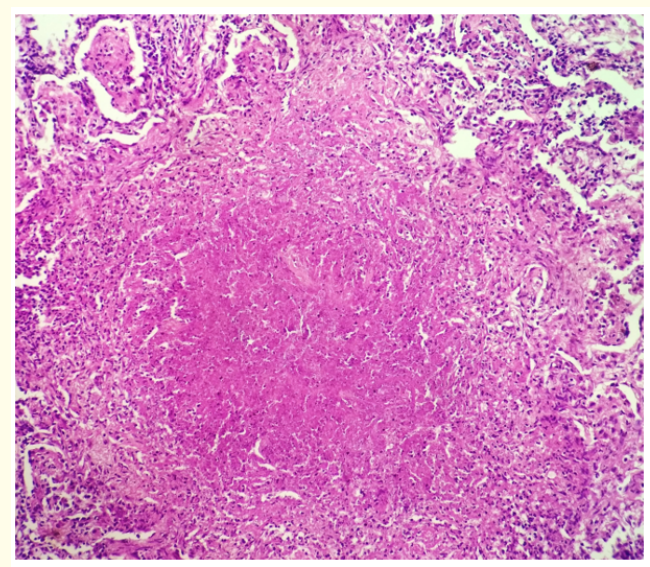

Figure 3: Microsections from lung revealing caseous necrosis, epithelioid cell granulomas and mononuclear cell infiltrate.

(HE;400x)

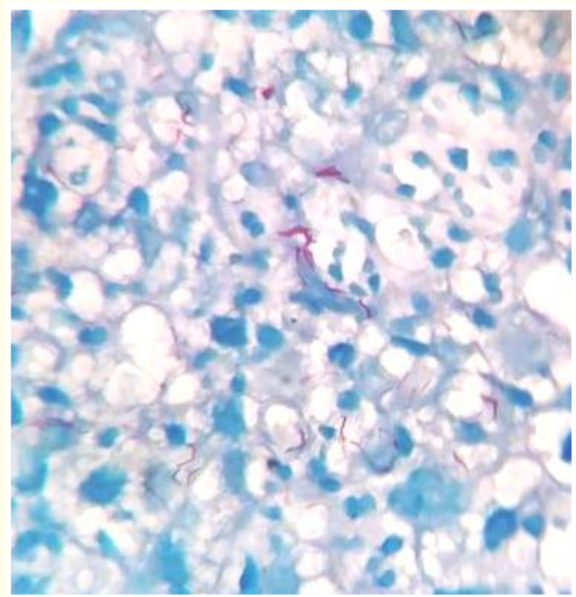

Figure 4: Acid fast bacilli in microsections from heart. (ZN stain;1000x)

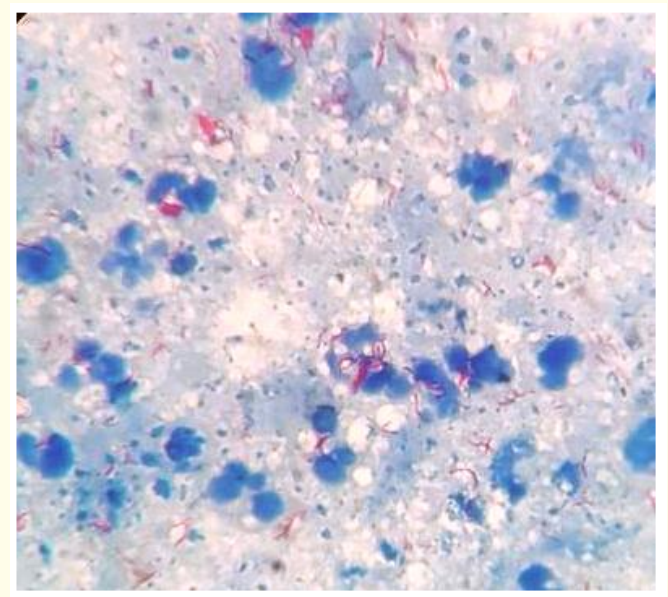

Figure 5: Acid fast bacilli in microsections from lung. (ZN stain;1000x).

\section{Discussion}

Tuberculous myocarditis is a rare disease of the heart characterised by the presence of epithelioid granulomas with caseous necrosis and chronic inflammatory cells. Tuberculosis and sarcoidosis are the most commonly reported causes of granulomatous myocarditis with sarcoidosis granulomas being non-caseating. Tubercular myocarditis can remain clinically asymptomatic with a diagnosis being made only at autopsy [7]. The delayed diagnosis is due to non-specific symptomatology including fever, night sweats, fatigue, weight loss, cachexia, cough, chest pain, breathlessness.

Four organs are considered to be resistant to tuberculosisheart, thyroid, pancreas and skeletal muscle. The reasons for heart being resistant are not known. However it has been suggested that constant movement of myocardium is not conducive to lodgement of tuberculi bacilli [2]. 
Pathological changes of tuberculous pericarditis are due to immune response to $\mathrm{M}$. tuberculosis bacilli penetrating the pericardium. Protein antigens of bacillus induce delayed hypersensitivity responses by stimulating lymphocytes to release lymphokines that activate macrophages and induce granuloma formation [8].

Milliary tuberculosis is one of the many lethal consequences of tuberculosis. In the present case, lung and liver were involved along with myocardium and pericardium. Milliary tuberculosis is caused due to lymphohematogenous spread of the infection from primary focus. There is dehiscence of mycobacterium rich inflammatory focus into blood vessels with dissemination of bacilli into blood stream and seeding into non- pulmonary sites. Predisposing factors for the development of this condition include advanced age, immunosuppression in cases of HIV, chronic renal failure, organ transplantation or corticosteroid therapy [9-12].

The common sites are the right atrium and left ventricle. Right atrial involvement is considered to be related to frequent involvement of the right mediastinal lymph node [13]. The three histologic patterns of myocardial tuberculosis are nodular, miliary, and diffuse infiltrative pattern [14].Nodular pattern shows pea to egg sized lesions with central caseation usually affecting the right side of the heart, especially the right atrium. Miliary pattern is seen in cases of generalized military disease. In diffuse infiltrative pattern there is diffuse infiltration of the myocardium by granulation tissue, giant cells and lymphocytes; this variant is uncommon and is usually associated with tuberculous pericarditis.

The differential diagnosis of myocardial tuberculosis includes granulomatous diseases such as sarcoidosis, idiopathic granulomatous and giant-cell myocarditis; syphilitic gummata, fungal infection, rheumatic fever and metastatic tumors containing giant cells, e.g., Hodgkin disease and osteosarcoma [14].

The diagnosis of myopericarditis require (1) culture of either pericardial tissueor fluid specimens that was positive for Mycobacterium tuberculosis, (2) granulomas and acid-fast bacilli (AFB) seen on histopathological examination of heart, or (3) granulomas in the tissue and a positive culture for M. tuberculosis from another site [15].

The demonstration of acid-fast bacilli within foci of necrotizing granulomatous inflammation, as seen in our case, is rare $[3,6,16]$. However, technologic advances in molecular biology allow identi- fication of the M. tuberculosis DNA complex in cases of suspected myocardial tuberculosis. The polymerase chain reaction can be performed on myocardial resection material for identification of $M$. tuberculosis-specific genes [17].

Tuberculosis is increasing at a faster rate and lack of treatment leads to its dissemination into other organs including heart. The diagnosis of such cases is missed or delayed due to either absence of specific symptoms or lack of investigations in patients for the suspicious symptoms.

\section{Conclusion}

Curbing the uprise of tuberculosis is possible if diagnosis and treatment for tuberculosis is started in time and completed with full compliance. The fatal rate and transmission rate is high if disease remains undiagnosed and such cases are thus a threat to population. In the era of medical research patients with curable diseases like tuberculosis instigate the need for early diagnosis. Death of patient can be averted with awareness and treatment. Tuberculosis is a major problem in developing countries and surveillance is needed to control its spread.

\section{Bibliography}

1. Agarwal R., et al. "Tuberculous dilated cardiomyopathy: an under-recognized entity?". BMC Infectious Disease 5 (2005): 29.

2. Chan ACL and Dickens P. "Tuberculous myocarditis presenting as sudden cardiac death". Forensic Science International 57.1 (1992): 45-50.

3. Rose AG. "Cardiac tuberculosis: a study of 19 patients". Archives of Pathology and Laboratory Medicine 111.5 (1987): 422-426.

4. Agarwal N and Sharma SK. "Concomitant endobronchial tuberculosis, myocarditis and congestive heart failure". Indian Journal of Tuberculosis 47 (2000): 169.

5. Wallis PJW., et al. "Sudden death due to myocardial tuberculosis". Thorax 39.2 (1984): 155-156.

6. Behr G., et al. "Myocardial tuberculosis". British medical journal 1.6066 (1977): 951.

7. Bali HK., et al. "Myocardial tuberculosis presenting as restrictive cardiomyopathy". American Heart Journal 120.3 (1990): 703-706. 
8. Burgess LJ., et al. "Cytokine production in patients with tuberculous pericarditis". International Journal of Tuberculosis and Lung Disease 6.5 (2002): 439-446.

9. Sharma SK., et al. "Miliary tuberculosis: new insights into an old disease". The Lancet Infectious Diseases 5.7 (2005): 415430 .

10. Tajiri T., et al. "Autopsy Cases of MiliaryTuberculosis: ClinicopathologicFeaturesIncluding Background Factors". Journal of Nippon Medical School 78.5 (2011): 305-311.

11. Abi-Fadel F and Gupta K. "Acute respiratorydistress syndrome withmiliarytuberculosis: a fatal Combination". Journal of Thoracic Disease 5.1 (2013):1-4.

12. Singh R., et al. "Generalised non-reactivetuberculosis: aclinicopathologicalstudy of four patients". Thorax 44.11 (1989): 952-955.

13. Kapoor OP., et al. "Tuberculoma of the heart: report of 9 cases". American Heart Journal 86.3 (1973): 334-340.

14. Dada MA., et al. "Sudden Death Caused by Myocardial Tuberculosis: Case Report and Review of the Literature". The American Journal of Forensic Medicine and Pathology 21.4 (2000): 385-388.

15. Trautner BW and Darouiche RO. "Tuberculous Pericarditis: Optimal diagnosis and management". Clinical Infectious Diseases 33.7 (2001): 954-961.

16. Halim MA., et al. "Myocardial tuberculoma with rupture and pseudoaneurysm formation: successful surgical treatment". British Heart Journal 54.6 (1985): 603-604.

17. Baretti R., et al. "Submitral left ventricular tuberculoma". The Annals of Thoracic Surgery 60.1 (1995): 181-182.

Volume 3 Issue 8 August 2019

(C) All rights are reserved by Sucheta., et al. 